\title{
Ultrasound definition of tendon damage in patients with rheumatoid arthritis. Results of a OMERACT consensus-based ultrasound score focussing on the diagnostic reliability
}

\author{
George A W Bruyn, ${ }^{1}$ Petra Hanova, ${ }^{2}$ Annamaria lagnocco, ${ }^{3}$ \\ Maria-Antonietta d'Agostino, ${ }^{4}$ Ingrid Möller, ${ }_{1}^{5}$ Lene Terslev, ${ }^{6}$ Marina Backhaus, ${ }^{7}$ \\ Peter V Balint, ${ }^{8}$ Emilio Filippucci, ${ }^{9}$ Paul Baudoin, ${ }^{1}$ Richard van Vugt, ${ }^{10}$ \\ Carlos Pineda, ${ }^{11}$ Richard Wakefield, ${ }^{12}$ Jesus Garrido, ${ }^{13}$ Ondrej Pecha, ${ }^{14}$ \\ Esperanza Naredo, ${ }^{15}$ on behalf of the OMERACT Ultrasound Task Force
}

Handling editor Tore K Kvien For numbered affiliations see end of article.

\section{Correspondence to} George A W Bruyn, Department of Rheumatology, MC Groep Hospitals, Lelystad 8333 AA, The Netherlands; gawbruyn@wxs.nl

Received 10 March 2013 Revised 20 May 2013 Accepted 28 June 2013 Published Online First 12 August 2013
CrossMark

To cite: Bruyn GAW,

Hanova $\mathrm{P}$, lagnocco $\mathrm{A}$, et al.

Ann Rheum Dis

2014;73:1929-1934.

\section{ABSTRACT}

Objective To develop the first ultrasound scoring system of tendon damage in rheumatoid arthritis (RA) and assess its intraobserver and interobserver reliability. Methods We conducted a Delphi study on ultrasounddefined tendon damage and ultrasound scoring system of tendon damage in RA among 35 international rheumatologists with experience in musculoskeletal ultrasound. Twelve patients with RA were included and assessed twice by 12 rheumatologists-sonographers. Ultrasound examination for tendon damage in B mode of five wrist extensor compartments (extensor carpi radialis brevis and longus; extensor pollicis longus; extensor digitorum communis; extensor digiti minimi; extensor carpi ulnaris) and one ankle tendon (tibialis posterior) was performed blindly, independently and bilaterally in each patient. Intraobserver and interobserver reliability were calculated by $\kappa$ coefficients.

Results A three-grade semiquantitative scoring system was agreed for scoring tendon damage in B mode. The mean intraobserver reliability for tendon damage scoring was excellent ( $\kappa$ value 0.91 ). The mean interobserver reliability assessment showed good $\kappa$ values ( $\kappa$ value 0.75). The most reliable were the extensor digiti minimi, the extensor carpi ulnaris, and the tibialis posterior tendons. An ultrasound reference image atlas of tenosynovitis and tendon damage was also developed. Conclusions Ultrasound is a reproducible tool for evaluating tendon damage in RA. This study strongly supports a new reliable ultrasound scoring system for tendon damage.

\section{INTRODUCTION}

Tenosynovitis is one of the key features of the clinical pattern in patients with rheumatoid arthritis (RA). ${ }^{1-3}$ Histologically, tenosynovitis exhibits similar features as joint synovitis, including hyperplasia of the synovial lining and infiltration of particular types of leukocytes, notably CD4 T cells and CD $68+$ macrophages. ${ }^{4}$ Longstanding tenosynovitis may result in tendon damage either by synovial proliferation or by bony attrition resulting in tendon rupture with consequent disability. ${ }^{5-7}$ The most common ruptures of the tendons of the hand involve the extensor pollicis longus (EPL) tendon and the extensor digiti minimi (EDM) tendon. It is assumed that partial tears in tendons progressively evolve into complete ruptures. Although clinical examination (CE) may disclose complete rupture, $\mathrm{CE}$ of partial tears is notoriously unreliable. ${ }^{5-7}$

Musculoskeletal ultrasound is a readily available, useful and versatile imaging modality with high patient acceptability. Musculoskeletal ultrasound has proven to be more accurate than $\mathrm{CE}$ in detecting synovitis and tenosynovitis. ${ }^{8}$

Despite these attractive features, the technique is still considered examiner-dependent and machinedependent. This opinion is based mainly on the fact that both acquisition and interpretation of ultrasound images determine the metric properties. Over the past decade, the Outcome Measures in Rheumatology in Clinical Trials (OMERACT) ultrasound Task Force, a group of interested international sonographers, has worked to address the metric qualities of musculoskeletal ultrasound in RA. ${ }^{9}{ }^{10}$ More recently, the Task Force has looked at the intraobserver and interobserver reliability of ultrasound for detecting and grading of greyscale tenosynovitis and tenosynovial power Doppler activity in patients with RA. ${ }^{11-13}$ The present study is an extension of these tendon studies and focuses on tendon damage.

The aim of the present study was threefold, that is, to achieve consensus on elementary lesions and definition of tendon damage in RA; to develop a novel ultrasound scoring system for tendon damage in B mode, and to assess the intraobserver and interobserver reliability of this scoring system for tendon damage in RA patients among rheumatologists with extensive experience in musculoskeletal ultrasonography (MSUS).

\section{PATIENTS AND METHODS}

\section{A two-step study}

The study was carried out in two steps. The first step consisted of a Delphi exercise, aiming to find agreement on ultrasound definitions of normal tendons, peritendinous structures, tenosynovitis and tendon damage in RA; furthermore, the Delphi 
exercise was done to reach consensus on the ultrasound grading of tenosynovitis and tendon damage in RA patients. Details on the methodology of the first step have previously been reported by Naredo et al. ${ }^{13}$

\section{ultrasound reliability assessment}

The first step of the study was followed by a two-day patientreliability exercise, which took place in Amsterdam, The Netherlands. Each day was divided in a morning and an afternoon session. The afternoon session was a repetition of the morning session in order to assess the intraobserver reliability.

\section{Patients}

Twelve patients with RA according to the American College of Rheumatology 1987 criteria $^{14}$ representing all degrees of disease activity (severe, moderate, low and remission as defined by DAS28) were recruited from the outpatient rheumatology clinic (MC Groep hospitals). Demographic and clinical data were recorded for all patients.

The 12 patients were equally divided over 2 days. Both wrists and ankles were studied for the ultrasound investigation. All patients were assessed twice, that is, during the morning and again in the afternoon. The local ethics committee approved the study and all patients gave written consent according to the Declaration of Helsinki.

\section{Ultrasonographers}

Twelve rheumatologists with extensive experience in ultrasound, that is, more than 10 years, participated in the present study.

\section{Tendons}

At the wrist, the following extensor tendons enclosed in a synovial sheath were selected: the second extensor compartment, that is, the extensor carpi radialis brevis and longus; the third, that is, the extensor pollicis longus (EPL); the fourth, that is, the extensor digitorum communis (EDC); the fifth, that is, the extensor digiti minimi (EDM); and the sixth, the extensor carpi ulnaris (ECU). At the ankle, the tibialis posterior tendon was included. Since flexor tendons at the wrist may show a high level of anisotropy making ultrasound evaluation of tendon damage difficult, they were not included in the ultrasound evaluation. $^{1213}$

\section{Ultrasonography}

Bilateral ultrasound investigation was performed with six Esaote ultrasound scanners (one Mylab 70 XVision and five Mylab Class C; Esaote, Genoa, Italy) by means of linear array transducers (6-18 MHz or 4-13 MHz). The B mode settings of each ultrasound machine were optimised and fixed. Dynamic investigation by flexion and extension of particular fingers was allowed to improve differentiation of tendon pathologies.

The 12 ultrasonographers independently, consecutively and blinded to the clinical data performed the ultrasound examination of the selected tendons and assessed tendon damage in B mode according to the agreed scoring system. The extensor tendons of the wrist were scanned from the level of Lister's tubercle downwards to the level of the extensor retinaculum; the tibialis posterior tendon was scanned from a level proximal to the medial malleolus to slightly distal of it. ${ }^{12}{ }^{13}$ Maximal scanning time was $15 \mathrm{~min}$ per patient. The scanning time included the time to fill out the scoring sheet.

\section{Atlas}

All members of the OMERACT US task group collected images which were used to develop an US reference image atlas of tenosynovitis and tendon damage.

\section{Statistical analysis}

Statistical analysis was performed with the software package SPSS, version 17.0. Normally distributed continuous data were summarised with means and SDs or 95\% CIs; non-normally distributed data were summarised with median and range.

Intra- and interobserver agreement was assessed by $\kappa$ coefficients. Cohen's $\kappa$ coefficient was calculated for intraobserver agreement, whereas Light's $\kappa$ was calculated for interobserver agreement. ${ }^{15} 16$ The comparison of the $\kappa s$ between first and second occasion was conducted using the Root Mean Square Difference index, and by the product-moment correlation coefficient. Basic statistics and interobserver reliability represented by the intraclass correlation coefficient (ICC) with 95\% CI were determined for each tendon compartment separately.

ICC and $\kappa$ values are comparable; $\kappa$ values were interpreted as follows: $0-0.20$ poor, $0.20-0.40$ fair, $0.40-0.60$ moderate, $0.60-0.80$ good and $0.80-1$ excellent agreement.

\section{RESULTS}

\section{Delphi process}

The results of the Delphi exercise regarding ultrasound definitions of normal tendons, anatomically related structures and tenosynovitis have been previously reported. ${ }^{13}$ Regarding the statements on tendon damage, there was group agreement on

(a)

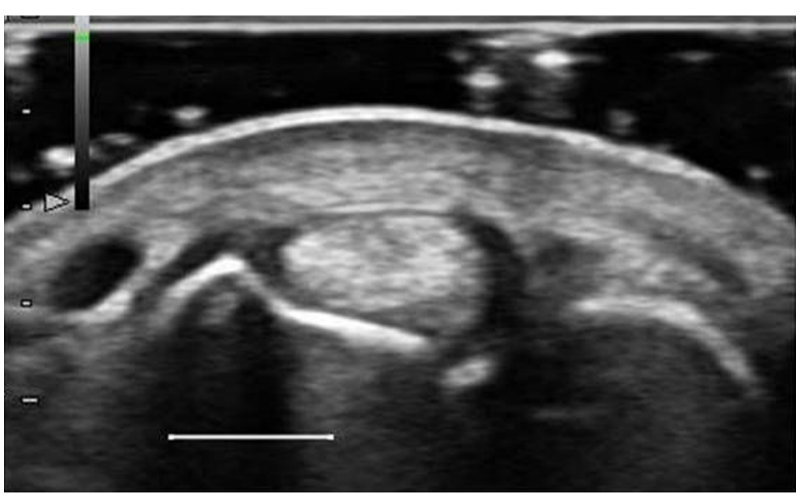

(b)

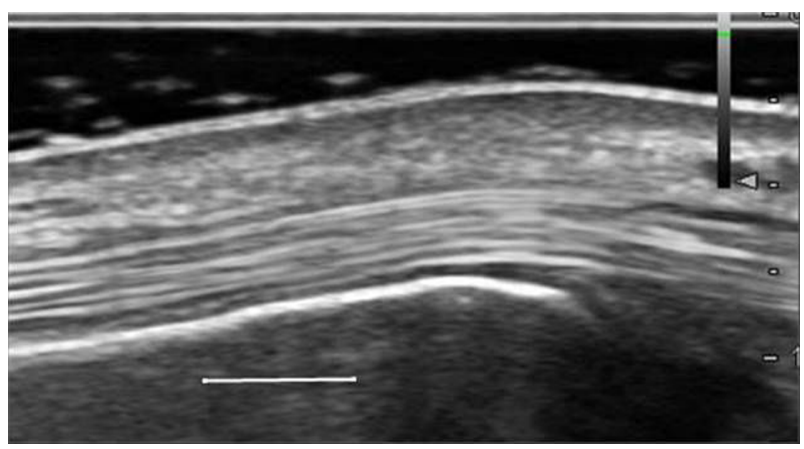

Figure 1 (A) Transverse scan in B mode of a normal extensor carpi ulnaris tendon, residing in its groove on the distal ulna. Tendon damage grade 0 . Dimension unit indicates $10 \mathrm{~mm}$. (B) Longitudinal scan in B mode of a normal extensor carpi ulnaris tendon. Tendon damage score grade 0 . Dimension unit indicates $10 \mathrm{~mm}$. 
(a)

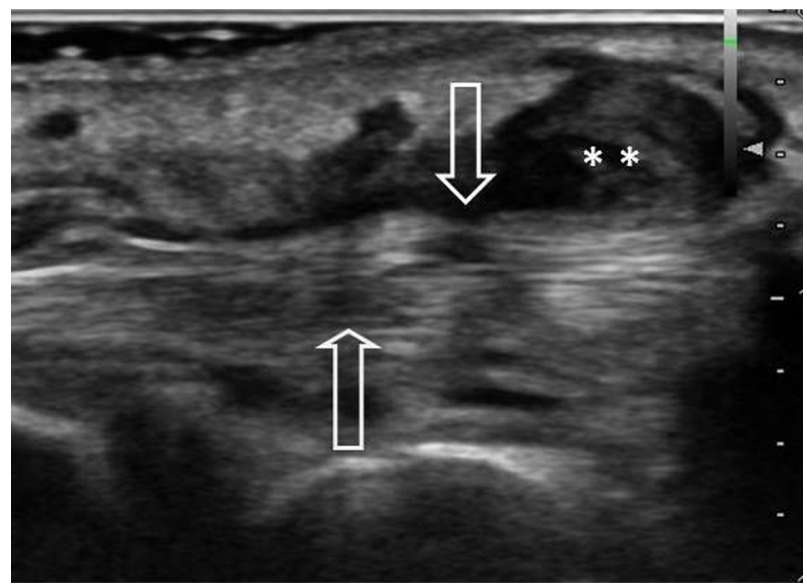

(b)

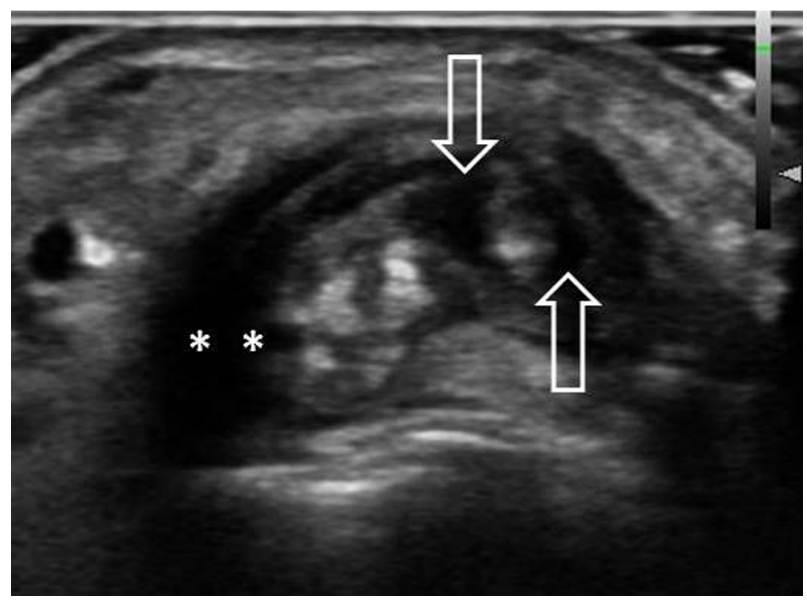

Figure 2 (A) Longitudinal scan of extensor carpi ulnaris tendon. Tendon damage score grade 1. Asterisks indicate an area of synovial proliferation within the tendon sheath, arrow point to partial rupture. (B) Transverse scan of extensor carpi ulnaris tendon. Tendon damage score grade 1. Asterisks indicate tenosynovitis, arrows point to partial rupture.

the definition of tendon damage and the scoring system after two rounds.

In particular, group agreement was achieved on the following items: tendon damage can be defined on B-mode as internal and/or peripheral focal tendon defect (ie, absence of fibres) in the region enclosed by tendon sheath, seen in two perpendicular planes; the grade of tendon damage should be assessed in both longitudinal and transverse planes; and, a four-grade semiquantitative scoring system (ie, grade 0 , normal; grade 1, minimal; grade 2, moderate; grade 3, severe) can be used to score tendon damage on B mode.

\section{Review of sent ultrasound images of tendons and consensus finding on scoring system}

Out of 28 consulted experts, 19 (68\%) sent a set of ultrasound images covering all grades of tendon damage to the organisers of the study (GAWB and EN). All the participants of the reliability exercise reviewed these images in a consensus meeting on the evening prior to the exercise. During this review process, it was noted that a four-grade semiquantitative scoring system did not work for most experts. Based on their opinion, the following scoring system for tendon damage in B mode was concurred: grade 0 , normal tendon; grade 1 , partial tendon damage (a)

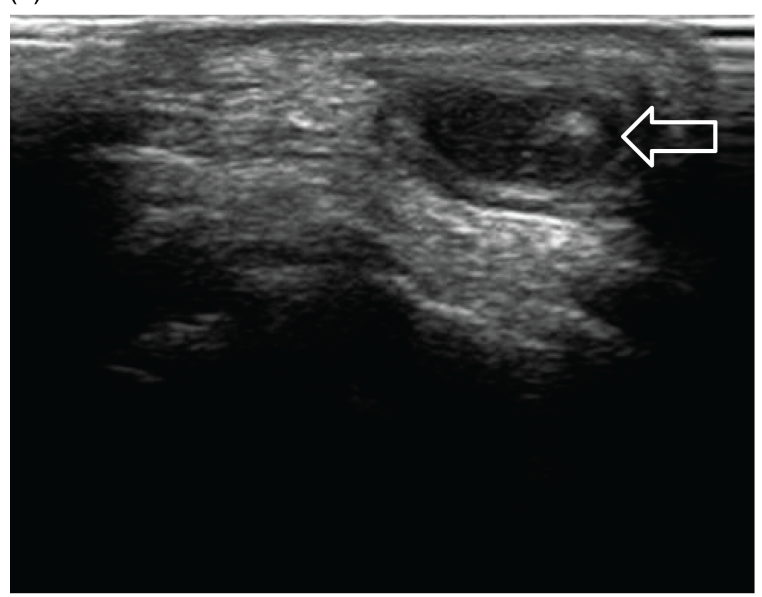

(b)

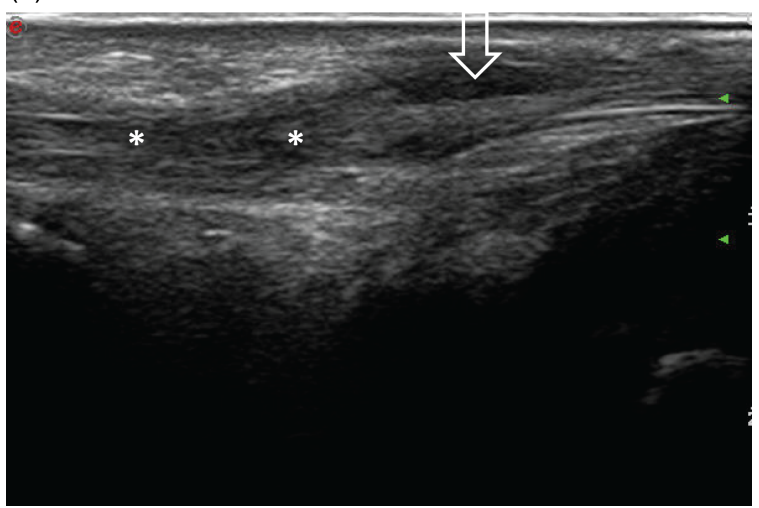

Figure 3 (A) Transverse sonogram showing a stump (arrow) of the completely ruptured extensor digiti minimi tendon. The stump is surrounded by fluid and synovial proliferation. (B) Longitudinal sonogram of the extensor digiti minimi tendon, showing the site of complete rupture $\left({ }^{* *}\right)$ and distension of the tendon sheath due to fluid and synovial proliferation (arrow).

Table 1 Demographical, disease-related characteristics and ultrasound grading

\begin{tabular}{lcl}
\hline Characteristics & $\mathbf{n}(\%)$ & Median, range \\
\hline Number of patients & $12(100)$ & - \\
Gender (F/M) & $7 / 5(58 / 42)$ & - \\
Age (years), median and range & & $66.5,36-74$ \\
Disease duration (months) & - & $76,12-104$ \\
Rheumatoid factor (RF)+ & $8(67)$ & - \\
Anti CCP+ & $5(42)$ & - \\
RF+ anti CCP+ & $3(25)$ & - \\
RF- anti CCP- & $2(17)$ & - \\
DAS 28 & - & $3.79,2.41-5.6$ \\
Remission (DAS 28<2.6) & $3(25)$ & - \\
Low disease activity (DAS 28<3.2) & $4(33)$ & - \\
Moderate disease activity DAS 28<5.2) & $3(25)$ & - \\
High disease activity (DAS 28 $>5.2)$ & $2(17)$ & - \\
Current use of anti-TNFa agents & $7(58)$ & \\
Erosive disease & $8(67)$ & \\
Cumulative prednisone dose (g) & & $19.1,0-30.5$ \\
Total vdHSS & & $25,4-44$ \\
Grade 0 tendon damage & $2652(76.7)$ \\
Grade 1 tendon damage & $727(21)$ \\
Grade 2 tendon damage & $77(2.3)$ \\
\hline CCP, anti-cyclic citrullinated peptide. & & \\
\end{tabular}

CCP, anti-cyclic citrullinated peptide. 
Table 2 Absolute frequencies and percentages of tendon damage according to tendon compartment

\begin{tabular}{lcccc}
\hline Compartment & Explored & Grade $\mathbf{0}$ & Grade 1, 2 & Prevalence* $^{*}$ \\
\hline 2R & 288 & 261 & 27 & 9.4 \\
3R & 288 & 243 & 45 & 15.6 \\
4R & 288 & 249 & 39 & 13.5 \\
5R & 288 & 261 & 27 & 9.4 \\
6R & 288 & 147 & 141 & 49.0 \\
Tib R & 288 & 225 & 63 & 21.9 \\
2L & 288 & 265 & 23 & 8.0 \\
3L & 288 & 283 & 5 & 1.7 \\
4L & 288 & 280 & 8 & 2.8 \\
5L & 288 & 183 & 105 & 36.5 \\
6L & 288 & 112 & 176 & 61.1 \\
Tib L & 288 & 143 & 145 & 50.3 \\
Total & 3456 & 2652 & 804 & $23.3 \dagger$ \\
\hline
\end{tabular}

*Prevalence is expressed as the percentage (grade 1 and grade 2 ) tendon damage found in the particular compartment ( $\mathrm{N}=288$ investigations per compartment). tDenotes the mean prevalence of tendon damage considering 804 tendon lesions among 3456 investigations. The median prevalence is $13.5 \%$, range, $1.7-61.1 \%$. $2 \mathrm{R}$ denotes second compartment of the right hand, Tib $L$ means tibialis posterior of the left ankle. The prevalence of findings in $3 \mathrm{~L}$ and $4 \mathrm{~L}$ is very low.

seen in two orthogonal planes, and grade 2, complete tendon rupture seen in two orthogonal planes. Greyscale examples of tendon damage are shown in figures $1-3$.

\section{Patient characteristics}

The demographics and disease-related characteristics of the patients with RA are summarised in table 1.

\section{Prevalence of ultrasound abnormalities}

Overall, 3456 tendon compartments were assessed by ultrasound in B mode (144 per investigator). Of these, 804 ultrasound investigations showed either a grade 1 or a grade 2 tendon lesion (23\%). The prevalence of lesions per tendon compartment is shown in table 2 .

\section{Intraobserver and interobserver agreement}

Table 3 shows the $\kappa$ coefficient estimates of interobserver agreement calculated for pairs of investigators at first and second (italic) occasion. Intraobserver agreement is shown as bold numbers on the diagonal line. Means are calculated below the table.

In table 4, the interobserver reliability of the tested scoring system within particular compartments is estimated.

\section{Atlas}

GS and PD US images of tendon lesions were collected into a US reference image atlas. The reference images include US images of tenosynovitis and tendon damage of various grades affecting tendons frequently involved in RA. Multiple examples covering semiquantitative grades of tenosynovitis (0-3) and tendon damage (0-2) are shown in the online supplementary material. In addition to the typical images, the atlas comprises a series of challenging Doppler images. With the guidance of the reference images displayed in this atlas, US scans of tendon abnormalities in RA can easily be scored for various grades of tenosynovitis and tendon damage both in clinical practice and in research trials (see online supplementary material).

\section{DISCUSSION}

To our knowledge, the present exercise is the first multiobserver study that assesses the reproducibility of ultrasound in scoring tendon damage in patients with RA. The results show a high intraobserver and interobserver reliability among experienced rheumatology ultrasonographers. The findings may be relevant for both daily clinical practice and trials. As yet, no ultrasound studies have assessed the grading of tendon damage in RA; a reliable imaging scoring system may be used to identify and follow-up tendons at risk of rupture. Furthermore, a ultrasound scoring system for tendon disease may serve as an imaging biomarker for clinical drug trials.

Only a few studies have looked at the ultrasound assessment of tendon damage in RA, observing a wide variability. Filippucci and colleagues found partial tendon tears in $12 \%$ and complete tears in $3 \%$ in a cross-sectional analysis of 90 patients with RA. ${ }^{17}$ Micu and colleagues found tendon damage in over $50 \%$

Table 3 Cohen's and Light's $\kappa$ estimates of intraobserver and interobserver agreement on the first occasion and second occasion; both days analysed together

\begin{tabular}{lllllllllllll}
\hline Rater & $\mathbf{1}$ & $\mathbf{2}$ & $\mathbf{3}$ & $\mathbf{4}$ & $\mathbf{5}$ & $\mathbf{6}$ & $\mathbf{7}$ & $\mathbf{8}$ & $\mathbf{9}$ & $\mathbf{1 0}$ & $\mathbf{1 1}$ \\
\hline 1 & $\mathbf{0 . 9 8 3}$ & 0.685 & 0.726 & 0.679 & 0.632 & 0.738 & 0.719 & 0.623 & 0.776 & 0.680 & 0.722 & 0.722 \\
2 & 0.689 & $\mathbf{0 . 9 3 9}$ & 0.836 & 0.702 & 0.730 & 0.732 & 0.748 & 0.719 & 0.766 & 0.741 & 0.916 & 0.789 \\
3 & 0.779 & 0.821 & 0.920 & 0.670 & 0.858 & 0.699 & 0.829 & 0.686 & 0.771 & 0.664 & 0.836 & 0.712 \\
4 & 0.782 & 0.708 & 0.803 & 0.867 & 0.686 & 0.652 & 0.667 & 0.562 & 0.679 & 0.656 & 0.702 & 0.742 \\
5 & 0.699 & 0.774 & 0.867 & 0.757 & 0.885 & 0.639 & 0.770 & 0.583 & 0.747 & 0.680 & 0.730 & 0.730 \\
6 & 0.786 & 0.829 & 0.808 & 0.811 & 0.798 & 0.891 & 0.694 & 0.630 & 0.710 & 0.570 & 0.732 & 0.732 \\
7 & 0.730 & 0.769 & 0.903 & 0.752 & 0.852 & 0.794 & 0.963 & 0.682 & 0.765 & 0.664 & 0.748 & 0.709 \\
8 & 0.616 & 0.686 & 0.702 & 0.630 & 0.661 & 0.600 & 0.692 & 0.825 & 0.696 & 0.631 & 0.799 & 0.639 \\
9 & 0.816 & 0.821 & 0.920 & 0.843 & 0.829 & 0.846 & 0.864 & 0.702 & 0.938 & 0.716 & 0.808 & 0.766 \\
10 & 0.779 & 0.742 & 0.799 & 0.725 & 0.715 & 0.731 & 0.747 & 0.623 & 0.839 & 0.874 & 0.741 & 0.784 \\
11 & 0.795 & 0.799 & 0.858 & 0.782 & 0.808 & 0.825 & 0.804 & 0.679 & 0.898 & 0.777 & 0.854 & 0.747 \\
12 & 0.819 & 0.786 & 0.843 & 0.807 & 0.757 & 0.849 & 0.790 & 0.669 & 0.882 & 0.764 & 0.782 & 0.920 \\
\hline
\end{tabular}

Mean inter-rater Light's $\kappa$ value (both occasions) $=0.746(S D \pm 0.075)$

Mean intra-rater Cohen's $\kappa$ value $=0.905(S D \pm 0.045)$ (on diagonal, bold entries).

$\mathrm{r} 1,2=0.623, \mathrm{RMSD}=0.084$.

Note. Coefficients in the lower left triangle belong to the first occasion; coefficients in the upper right triangle (italic) belong to the second occasion; RMSD, Root Mean Square

Difference; r1,2, correlation between the first and the second occasion; based on dichotomised dataset. 
Table 4 Descriptives and interobserver reliability (ICC) of the tested scoring system within particular tendon compartments

\begin{tabular}{|c|c|c|c|c|c|}
\hline $\begin{array}{l}\text { Tendon } \\
\text { compartment }\end{array}$ & Mean & SD & ICC & $95 \% \mathrm{Cl}$ & $\begin{array}{l}p \\
\text { Value }\end{array}$ \\
\hline $2 \mathrm{R}$ & 0.094 & 0.283 & 0.978 & (0.954 to 0.993$)$ & $<0.001$ \\
\hline $2 \mathrm{~L}$ & 0.118 & 0.301 & 0.898 & (0.786 to 0.965$)$ & $<0.001$ \\
\hline $3 R$ & 0.222 & 0.551 & 0.977 & (0.953 to 0.992$)$ & $<0.001$ \\
\hline $3 \mathrm{~L}$ & 0.035 & 0.174 & 0.425 & $(-0.208$ to 0.805$)$ & 0.073 \\
\hline $4 \mathrm{R}$ & 0.163 & 0.413 & 0.903 & (0.797 to 0.967$)$ & $<0.001$ \\
\hline $4 \mathrm{~L}$ & 0.101 & 0.072 & 0.428 & $(-0.202$ to 0.806$)$ & 0.071 \\
\hline $5 R$ & 0.094 & 0.289 & 0.961 & (0.919 to 0.987$)$ & $<0.001$ \\
\hline $5 \mathrm{~L}$ & 0.163 & 0.359 & 0.966 & (0.929 to 0.988 ) & $<0.001$ \\
\hline $6 \mathrm{R}$ & 0.493 & 0.491 & 0.973 & (0.943 to 0.991$)$ & $<0.001$ \\
\hline $6 \mathrm{~L}$ & 0.795 & 0.688 & 0.991 & (0.982 to 0.997 ) & $<0.001$ \\
\hline Tib R & 0.368 & 0.477 & 0.969 & (0.935 to 0.989$)$ & $<0.001$ \\
\hline Tib L & 0.507 & 0.502 & 0.986 & (0.971 to 0.995$)$ & $<0.001$ \\
\hline
\end{tabular}

of their patients. ${ }^{18}$ Our work reveals a partial rupture in $21 \%$ and a complete rupture in $2 \%$.

While the Delphi exercise resulted in a four-grade tendon damage score, the common opinion of the rheumatologists attending the patient exercise meeting was that a three-grade semiquantitative score (normal, partial and complete rupture), was more practical. As the meeting consensus was final, we used this score for the patient exercise.

Very few observers finally scored a complete rupture, that is, grade 2. This low prevalence of complete tendon rupture is probably due to the current treat-to-target strategy and the effectiveness of the new treatments of RA, that is, biological therapy. ${ }^{19}$ This finding was taken into account by the statistics processing and data were dichotomised where 0 was a completely normal tendon and 1 represented damage.

Some differences in reliability of scoring of tendon damage within particular compartments were found. This is probably due to various difficulties of investigations of particular tendons. The most reliable tendons were the ECU and tibialis posterior tendon-both non-splitting and relatively thick and straight-running tendons. One other very reliable tendon was the EDM. The ECU proved also to be the most often damaged tendon of all tendons investigated in the study. All the above mentioned tendons have been reported to be frequently involved in RA. ${ }^{5} 2021$ The presence of involvement of the ECU predicts the development of erosions ${ }^{21}$; therefore, the outcome of this study may support the use of the ECU as a reliable biomarker.

The least reliable tendons were EDC and EPL, probably due to splitting in some finger extensor tendons which may cause difficulties in interpretation of tendon damage, especially in transverse view of the EDC tendon. The EPL tendon can be difficult to follow in its course while crossing other tendons.

There are limitations inherent in our study. First, only 12 patients were assessed. However, similar numbers of patients have been assessed in other multiexaminer reliability studies for feasibility reasons. The difference in reliability noted between the right and left compartments three and four is related to the small prevalence of positive findings on the left side compared with the right, rather than true differences among observers in scoring lesions. Second, the lack of a gold standard, for example, MRI or surgery prevented to determine the true prevalence of tendon damage lesions. However, a concurrent validity study showed comparative accuracy in diagnosing tendon damage between ultrasound and MRI. ${ }^{22}$ Additionally, this was not a validity but a reliability study. Finally, the rheumatologists involved were all expert in ultrasonography. Thus, it is not taken for granted that these reliability results can be extrapolated to a population of less experienced rheumatologists. It is reassuring, however, that the broad US7 experience in Germany has revealed a good correlation between experts and less experienced rheumatologists. ${ }^{23}$

A strength of this study is the inclusion of the acquisition phase of ultrasound images. Another strength is that the reliability was assessed separately for particular tendon compartments.

In conclusion, the present study suggests that rheumatologists-ultrasonographers can have a high reliability in their performance of ultrasound assessment of 12 target tendon compartments in RA patients, with the best scores in the EDM, ECU and tibialis posterior tendons.

\section{Author affiliations}

${ }^{1}$ Rheumatology Department, MC Groep Hospitals, Lelystad, The Netherlands ${ }^{2}$ Department of Rheumatology, Institute of Rheumatology, Prague, Czech Republic ${ }^{3}$ Department of Rheumatology, Sapienza Università di Roma, Rome, Italy ${ }^{4}$ Department of Rheumatology, Université Paris Ouest-Versailles-Saint Quentin en Yvelines, Hôpital Ambroise Paré, APHP, Boulogne-Billancourt, France

${ }^{5}$ Department of Rheumatology, Instituto Poal, Barcelona, Spain

${ }^{6}$ Department of Rheumatology, Copenhagen University Hospital at Glostrup, Copenhagen, Denmark

${ }^{7}$ Department of Rheumatology, Charite University Hospital, Berlin, Germany ${ }^{8}$ Department of Rheumatology, National Institute of Rheumatology and Physiotherapy, Budapest, Hungary

${ }^{9}$ Department of Rheumatology, Clinica Reumatologica, Universitá Politecnica delle Marche, Jesi, Ancona, Italy

${ }^{10}$ Department of Rheumatology, VU Medisch Centrum, Amsterdam, The Netherlands

${ }^{11}$ Department of Rheumatology, National Institute of Rehabilitation, Mexico City,

Mexico

${ }^{12}$ Academic Unit of Musculoskeletal Disease, University of Leeds, Leeds, UK

${ }^{13}$ Department of Social Psychology and Methodology, Faculty of Psychology,

Autonoma University, Madrid, Spain

${ }^{14}$ Technology Centre ASCR, Prague, Czech Republic

${ }^{15}$ Department of Rheumatology, Hospital General Universitario Gregorio Marañón, Madrid, Spain

Correction notice This article has been corrected since it was published Online First. Figures $3 \mathrm{~A}$ and $3 \mathrm{~B}$ have been replaced and the legend amended. In addition, the grant numbers have been included in the Funding section.

Acknowledgements We thank the patients who participated in the reliability session. We are grateful to Esaote Netherlands BV for providing the ultrasound machines. We express our gratitude to Ria de Kort and Marian de Waal for logistical support.

Collaborators OMERACT Ultrasound Task Force members: Sibel Aydin, Artur Bachta, Paz Collado, Cristina Estrach, Jane E Freeston, Frederique Gandjbakhch, Marwin Gutierrez, Hilde B Hammer, Kei Ikeda, Frederick Joshua, Sandrine Jousse-Joulin, David Kane, Helen I. Keen, Juhani M Koski, Peter Mandl, Zunaid Karim, Wolfgang A Schmidt, Nanno Swen, Philip G Conaghan.

Contributors Study design: EN, GAWB, M-AD. Acquisition of data: ENaredo, $M-A D, P H, I M, P V B, E F, A l, C P, L T, M B, P B, R v V$, RW, GAWB. Analysis and interpretation of data: GAWB, PH, JG, OP, EN. Manuscript: GAWB, EN. Statistical analysis: $\mathrm{PH}, \mathrm{OP}, \mathrm{JG}$

Funding Roche Netherlands BV provided funding for the reliability exercise. Roche Netherlands BV did not participate in the study design, data collection, data analysis, or writing of the manuscript. Supported by the project (Ministry of Health, (zech Republic) for conceptual development of research organization 023728 (Institute of Rheumatology) and by project No. NT12437.

Competing interests None.

Ethics approval MEC hospital approval.

Provenance and peer review Not commissioned; externally peer reviewed. 


\section{REFERENCES}

1 Boutry N, Larde A, Lapegue F, et al. Magnetic resonance imaging appearance of the hands and feet in patients with early rheumatoid arthritis. J Rheumatol 2003;30:671-9.

2 Eshed I, Feist E, Althoff CE, et al. Tenosynovitis of the flexor tendons of the hand detected by MRI: an early indicator of rheumatoid arthritis. Rheumatology 2009;48:887-91.

3 Wakefield RJ, O'Connor PJ, Conaghan PG, et al. Finger tendon disease in untreated early rheumatoid arthritis: a comparison of ultrasound and magnetic resonance imaging. Arthritis Rheum 2007;57:1158-64.

4 Kaibara N, Yamada H, Shuto I, et al. Comparative histopathological analysis between tenosynovitis and joint synovitis in rheumatoid arthritis. Histopathology 2008:52:856-64.

5 Straub LR, Wilson EH. Spontaneous rupture of extensor tendons in the hand associated with rheumatoid arthritis. J Bone Hand Surg 1956;38:1208-17.

6 Rubens DJ, Blebea JS, Totterman SMS, et al. Rheumatoid arthritis: evaluation of wrist extensor tendons with clinical examination versus MRI imaging: a preliminary report. Radiology 1993;187:831-8.

7 Rubin DA, Keeland JB, Kitay GS, et al. Flexor tendon tears in the hand: use of MR imaging to diagnose degree of injury in a cadaver model. Am J Roentgenol 1996;166:615-20.

8 Naredo E, Bonilla G, Gamero F, et al. Assessment of inflammatory activity in rheumatoid arthritis: a comparative study of clinical evaluation with grey-scale and power Doppler ultrasonography. Ann Rheum Dis 2005;64:375-81.

9 Naredo E, Wakefield RJ, lagnocco A, et al. The OMERACT Ultrasound Task Force. Summary of advances and priorities. J Rheumatol 2011;38:2063-7.

10 Joshua F, Lassere M, Bruyn GA, et al. Summary findings of a systematic review of the ultrasound assessment of synovitis. J Rheumatol 2007;34:839-44.

11 Alcalde M, D'Agostino MA, Bruyn GA, et al. A systematic literature review of ultrasound definitions, scoring systems and validity according to the OMERACT filter for tendon lesion in rheumatoid arthritis and other inflammatory joint disease. Rheumatology (Oxford) 2012;51:1246-60.
12 Bruyn GA, Möller I, Garrido J, et al. Reliability testing of tendon disease using two different scanning methods in patients with rheumatoid arthritis. First step towards an ultrasonography scoring index. Rheumatology (Oxford) 2012;51:1655-61.

13 Naredo E, D'Agostino MA, Wakefield RJ, et al. Reliability of a consensus-based ultrasound score for tenosynovitis in rheumatoid arthritis. Ann Rheum Dis 2013;72:1328-34.

14 Arnett FC, Edworthy SM, Bloch DA, et al. The American Rheumatism Association 1987 revised criteria for the classification of rheumatoid arthritis. Arthritis Rheum 1988;31:315-24.

15 Light RJ. Measures of response agreement for qualitative data: some generalizations and alternatives. Psychol Bull 1971;76:365-77.

16 Cohen JA. A coefficient of agreement for nominal scales. Educ Psychol Meas 1960;20:37-46

17 Filippucci E, Gabba A, Di Geso L, et al. Hand tendon involvement in rheumatoid arthritis: an ultrasound study. Sem Arthritis Rheum 2012;41:752-60.

18 Micu MC, Serra S, Fodor D, et al. Inter-observer reliability of ultrasound detection of tendon abnormalities at the wrist and ankle in patients with rheumatoid arthritis. Rheumatology (Oxford) 2011;50:1120-4.

19 Hammer HB, Kvien TK. Ultrasonography shows significant improvement in wrist and ankle tenosynovitis in rheumatoid arthritis patients treated with adalimumab. Scand J Rheumatol 2011;40:178-82.

20 Williamson L, Mowat A, Burge P. Screening for extensor rupture in rheumatoid arthritis. Rheumatology 2001;40:420-3.

21 Lillegraven S, Bøyesen P, Hammer HB, et al. Tenosynovitis of the extensor carpi ulnaris tendon predicts erosive progression in early rheumatoid arthritis. Ann Rheum Dis 2011;70:2049-50.

22 Swen WAA, Jacobs JWG, Hubach PC, et al. Comparison of sonography and magnetic resonance imaging for the diagnosis of partial tears of finger extensor tendons in rheumatoid arthritis. Rheumatology 2000;39:55-62.

23 Ohrndorf $\mathrm{S}$, Fischer $\mathrm{U}$, Kellner $\mathrm{H}$, et al. Reliability of the novel 7-joint ultrasound score: results from an inter- and intraobserver study performed by rheumatologists. Arthritis Care Res 2012;64:1238-43. 
Ultrasound definition of tendon damage in patients with rheumatoid arthritis. Results of a OMERACT consensus-based ultrasound score focussing on the diagnostic reliability

George A W Bruyn, Petra Hanova, Annamaria lagnocco, Maria-Antonietta d'Agostino, Ingrid Möller, Lene Terslev, Marina Backhaus, Peter V Balint, Emilio Filippucci, Paul Baudoin, Richard van Vugt, Carlos Pineda,

Richard Wakefield, Jesus Garrido, Ondrej Pecha and Esperanza Naredo

Ann Rheum Dis 2014 73: 1929-1934 originally published online August 12,2013

doi: 10.1136/annrheumdis-2013-203596

Updated information and services can be found at:

http://ard.bmj.com/content/73/11/1929

\section{Supplementary Material}

References

Email alerting service
These include:

Supplementary material can be found at:

http://ard.bmj.com/content/suppl/2014/09/16/annrheumdis-2013-2035 96.DC1.html

This article cites 23 articles, 12 of which you can access for free at: http://ard.bmj.com/content/73/11/1929\#BIBL

Receive free email alerts when new articles cite this article. Sign up in the box at the top right corner of the online article.

$\begin{array}{cc}\text { Topic } & \text { Articles on similar topics can be found in } \\ \text { Collections } & \text { Clinical diagnostic tests (1279) } \\ & \text { Radiology (1112) } \\ & \text { Radiology (diagnostics) (750) } \\ & \text { Connective tissue disease (4236) } \\ & \text { Degenerative joint disease (4622) } \\ & \text { Immunology (including allergy) (5120) } \\ & \text { Musculoskeletal syndromes (4931) } \\ & \text { Rheumatoid arthritis (3245) }\end{array}$

\section{Notes}

To request permissions go to:

http://group.bmj.com/group/rights-licensing/permissions

To order reprints go to:

http://journals.bmj.com/cgi/reprintform

To subscribe to BMJ go to:

http://group.bmj.com/subscribe/ 